\title{
THE APPLICATION OF NANOTECHNOLOGIES IN PRODUCING LIGHT INDUSTRIAL MATERIALS
}

\author{
Maryam Ghorbani \\ Master of Architecture Group, Khalkhal Branch, Islamic Azad University, Khalkhal, Iran \\ mgorbaniamoogin@yahoo.com \\ Hojjatollah Rashid Kalubar \\ Faculty Member, University of Mohaghegh Ardebili, Assistant professor of Islamic Azad University, \\ Ardabil, Iran
}

\begin{abstract}
In this paper, the application of nanotechnology in producing light and sustainable industrial materials has been discussed. Including the important efficiency of nanotechnology in the work construction of industrial materials was reducing energy consumption, the making speed and reducing the economic cost of the building. In this study, researcher with the main goal of access to the safe and sustainable work construction, by using new technology of materials, the application of technology used in materials was determined by introducing and studying its composite materials such Nano. Due to the nature of the objectives in this research, the research method has been librarian, analytical, software and observational. Research concepts were associated with architectural spaces in relation to creativity in innovation and discovery of new industrial materials for stability and flexibility of executive materials. According to check the carried out changes and obtained results of this research, the researcher's research findings with regard to preserving traditional architectural identity, has searched in lightweight of new industrial materials such as, sandwich panels, autoclave concrete, modularity, covering the monolith, poly stone, polystyrene and fiber concrete.
\end{abstract}

Keywords: Technology, Nano, Industrial, Sustainability, Technology.

\section{INTRODUCTION}

With an overview of the past of architecture, it can be noted that the architecture have been changes from ancient times until today. If you look to the architecture of age of Industrial revolution, these changes in production of prefabricated components, and transferring them to the workshop are appeared as building components and turn it into a building so that in the nineteenth century in a very short time, both materials and methods of work construction were changed. Building components are made in factories and they were assembled quickly after transported to the site (Helman, 2002). In the twentieth century the most important engagement of architects has been building making by industrial method. This not only means mass production of building components but also means making the whole building such as automobile production lines by using components such as bathroom, prefabricated kitchens and advanced technology to achieve the high standards (Helman, 2002). The correct approach of industrial production of building in different directions can be included long-term goals of policy makers of work construction industry. Work construction of building as industrial is of such important factors in increasing production and establishing a balance between supply and demand marketable. But yet, this factor has not been found fully its position in our country. Therefore, with progress and the need for national recognition, at first must change the point of view of engineering community and country planners in relation to this important principle and also led the public to its side. Until after this, resorting to technologies of building become more comfortable and more tangible (Esmaeili-e-denarati \& aliborz, 2012). 
In modern societies, new look to method of making the building requires using new materials. Although, the building technology and implementing method may be unknown in the use of these materials still. But recognition of new materials and explore their properties are effective help to feel a need of them in the community (Jodeiri-e-Abbasi \& Javanmard, 2012). Looking at the new events of technology, the effects of applied relationships and integration of new industrial materials with traditional materials has become clear in sustainable architecture. Of these, the suggested strategies become criterion for designers and architects researcher to cope with the current challenges in the industrialization that is the result of continuity of industrial productions of work construction. The carried out studies are coherent with introducing the most worthwhile building materials, with the importance of its applied affect in reducing the costs and quality of its implementation, for continuity and sustainable development of architecture. The impact of industrial materials technology in the formability and quality of execution, the impact of industrialization in reducing the economic costs of building, the interaction of traditional architecture with contemporary architecture, the industrial production continuity in structure and architecture are of topics that have considerable impact in the application of new ideas and help to improve the technical knowledge level of work construction.

\section{CONCEPT OF TECHNOLOGY}

Technology is a word that is recently entered to the contemporary literature, and despite the importance and its application expansion, there are many ambiguities. In the dictionary, McGraw Hill, has been described the technology as a knowledge and systematic action in industrial processes, but generally generalized to any other activity. Larousse dictionary defines technology as follows: technology is study of tools, practices and methods used in various fields of industry. In summary, technology is the main factor to convert natural resources to work and services. Technology in industry science is associated with another word that is named the technology, but of distinguish conceptually: Technology is a technique that is reagent of knowledge. Technique has several parts, for example "tool technique" is a technique that there is in objects. The "tool human" is pointed to the human technique. The "tool information" is referred to documents. The "tool institutions" is a technique that is crystallized in institutions. In fact, technology is the science of technique methodology or the cognition of tools for applying the technique. Also, it is checking the ways or techniques to achieve the technique. Technology is study of a technique, which has applied in various branches of industrial. In general, the technology can be defined as the systematic study of build and run techniques. Another word that clearly explains the technology is the concept of industrialization. Also, the industrialization is making system to the industrial production method or in other word is a modernized and advanced operation. For more details of the industrialization relationship with technology, the new building materials have been studied along with their structural combination and its effect on quality of making and run.

\section{APPLICATION OF NANOTECHNOLOGY IN NEW BUILDING MATERIALS}

For checking the application of technology in industrial materials, the need of understanding its details of structural components is necessary. For example, nanotechnology is one of the factors causing changes or shaping the material. Nanotechnology is the study of material particles at the atomic scale for their control. Nanotechnology is used in electronics, biology, genetics, aeronautics, process of building and even is used in energy studies. Nano is a Greek word meaning small and is used to determine the value of a billionth of a quantity, because an atom is about 10 Nanometer. This expression is used for the public study of atomic and molecular particles. The use of nanotechnology in materials exploitation and building materials has been great progress. These improvements can be observed in new materials of industrialization such as polystyrenes, concrete coverage and isolators or modular divider. Also, it can be observed in other technical industrial materials as follow:

\section{SANDWICH PANELS}

In fact, a sandwich panel consists of two main parts: I) it is the central core that is poor and usually large; II) the shells located on either side of the core that is strong and usually thin. The weak central core is often foam or a honeycomb material. The shells located on either side of the core are made of the glass fiber composites or natural fibers. This seemingly simple structure, that it is called to this name due to the seeming similarity with sandwich, shows super advantages and capabilities of itself. 
A sandwich structure has resistance much higher than its individual components and has the extraordinary lightness. Also has relatively low cost and can be quickly and easily used in construction. After pultrusion profiles and products prepared by open mold method, the sandwich structures are the most important usage of the composites in building industry (Figure 1).

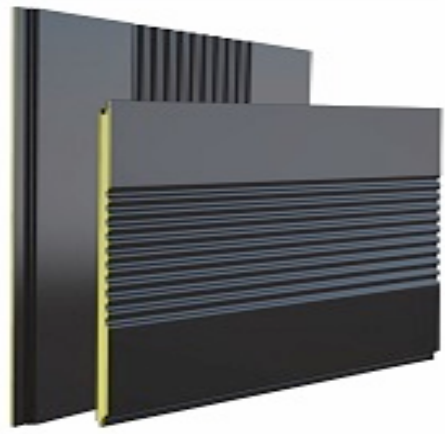

Figure 1. Plates image of sandwich panels

Although these panels are prepared through hand picking layered and by open mold method in the past, but today by the aid of machine processes, the speed and product quality has increased extraordinary. The same issue reduces the cost and increased welcoming these products. In addition to construction, the much use of sandwich panels can be seen in the aerospace industry, automobile, shipbuilding and so on (Jodeiri-e-Abbasi \& Javanmard, 2012).

\section{DETAILS OF THE STRUCTURE OF SANDWICH PANELS}

Sandwich panels (3D) is formed of a polystyrene layer with a thickness of at least four centimeter and two welded rebar networks on the both sides of this layer. The $30 \mathrm{~cm}$ model is recommended to select the width and height of the panels (the width $90-120-150 \mathrm{~cm}$ and a height of 270 and $300 \mathrm{~cm}$ ). The average weight of each page with size of $300 \times 150 \mathrm{~cm}$ was light with no concrete. It is easily portable and it can be installed by one worker. The action speed of installation is also impressive. The page resistance is perfect against the fire. The use of fire-resistant layer is recommended to improve it. With regard to the insulation layer of concrete, using these pages in addition to improving thermal and sound insulation properties of walls makes the building lightweight. Apart from reducing the volume of consumed materials, the building mass will reduce. By using these plates in parking, the building makes restrictions. Therefore, using hybrid system consisting of a steel structure with reinforced concrete and sandwich plates as separator is important on the requirement to create parking in the lower floors of buildings, s (Figure 2).

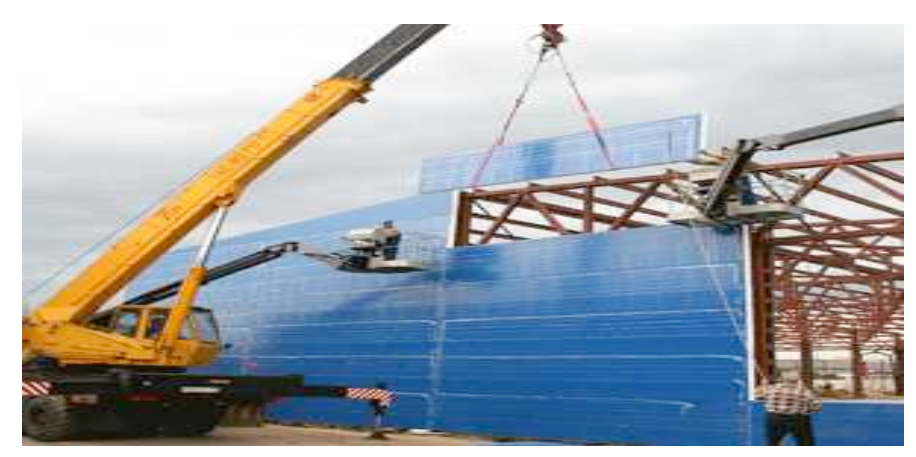

Figure 2. the image of combining steel structure with sandwich plates

According to the obtained information from European countries, the most buildings that have been implemented by this way are one or two floors. So, design and implementation of buildings with more 
floors requires special study and then, the study of design engineer must be answered the conditions of the valid regulations.

\section{ADVANTAGES OF SANDWICH PANELS}

1. The Lightweight of made walls of sandwich panels in comparison with other materials;

2. The speed and ease transport of sandwich panels in height;

3. The great resistance to shear forces caused by earthquake;

4. The insulation against heat, cold, humidity and noise;

5. The fire resistance due to the concrete layers on sides of sandwich panel;

6. The impermeability of building against insects;

7. The Ability to carry and use sandwich panels in impassable areas for buildings construction without a need for skilled workers;

8. Achieve to more useful space due to the small thickness of sandwich panels walls;

9. The freedom of action in various projects due to the flexibility of prefabricated components sandwich panels;

10. Reducing the cost of construction of the foundation and skeletons of high-rise buildings, due to low weight of roof components and wall sandwich panels;

11. Save on building air conditioning costs in summer or winter due to avoid heat exchange or cold exchange, and finally less energy consumption;

12. No relative influence of noise pollution and create comfort for building occupants in big cities

13. Remove horseshoe port in advanced sandwich panels

14. Transport capability at a lower cost; (For example, a trailer can carry about 1,000 square meters of sandwich panels).

15. No need for carving and destroying the walls and ceilings and resulting in no creation accumulated debris that saving the cost and the time.

\section{MODULARITY AND MODULAR DESIGN AS AN EFFECTIVE METHOD FOR DEVELOPING SUSTAINABLE HOUSING}

Modular design is a building construction method in which the run and how to make building is done by prefabricated materials or modular system. In the Scandinavian countries and the United States, owners and buyers are supported still in the event of increasing the cost higher than estimated cost. These prefabricated houses are shared widely (Carol Sta, 1988).

Types of modular systems or internal and external dividers of building throughout the world are as follows:

1. The Plate System; including flat plates in size of chart wall or the entire wall for separator or porter; (Fiqure3) 


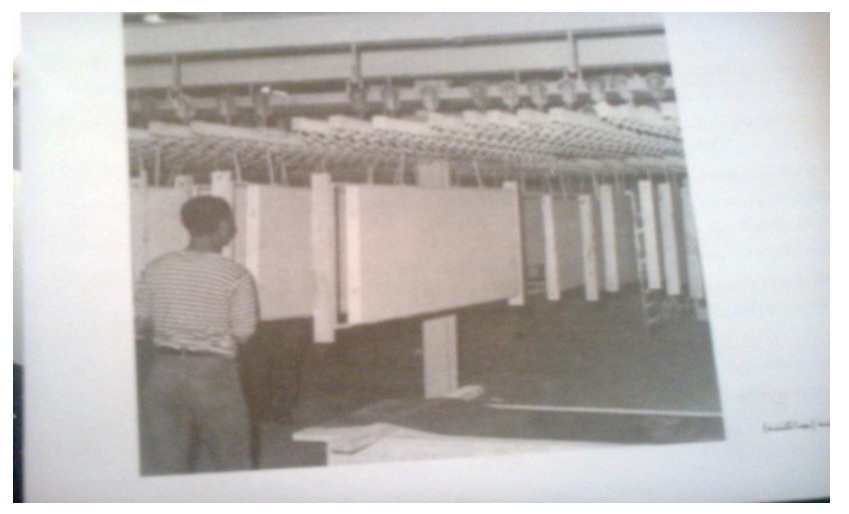

Figure 3. Picture separator Plate Systems

2. The frame system (skeletal) that gives the freight operation to the skeletal frame. separator non porter walls are used between them and in other words, are used from two different groups of elements; (Fiqure4)

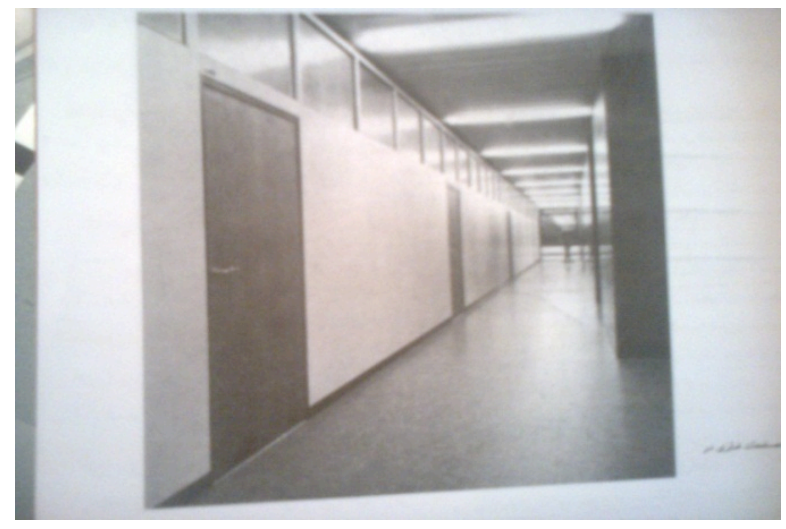

Figure 4. The factory images of divider elements

3. Volume or celled System is as different cells that are constructed as both porter and separator in the same size rooms (Carol Sta, 1988).

\section{GENESIS HISTORY OF MODULAR BUILDINGS}

The modular design is another solution that will has a great impact on reducing costs. The modular design can be appeared in the design of the building form, plan, or designing building components such as facade design and windows. The Carapanchel residential complex in Madrid is usage of modular design in the general form of a building. By using module, designers are looking to optimize the general structure of the building. The building skeleton is concrete that only is made by using a precise modulus for frame. Also, this industrial design is caused waste reduction materials and also, facilitates work construction and consequently reduces the time. The sum of these causes decrease the final cost of construction (Bashiri, 2012).

Modular design, as the name implies, is the building modularity. This thought means the produced prefabricated components that are related to the Seventeenth century. English residing in America used prefabricated walls that were composed of wooden frame, because they are easily portable and are placed inside the ship. They have short time to make housing; from the moment the ship arrives to cradle of modern civilization had a chance until the start of the cold season. So in the shortest time and with the least effort must have attempt to making shelter for selves. This emergency state led to genesis the American system that known as the wooden frame and was used to this day (Carol Sta, 
2000). In Europe, the thought of modular buildings were shaped in the late nineteenth century. The industrial revolution in its path with the genesis of new building materials such as steel girders and other metal products, steel and finally reinforced concrete were accelerated the creating of industrial and modular buildings. Labor shortages after World War II were the real stimulus to accelerate the implementation of modular buildings in different countries (Carol Sta, 2000).

\section{ASSESSMENT OF CHANGES IN THE COST OF BUILDING WITH MODULAR DESIGN}

Human casualties and material destruction caused by World War II were two main reasons of development of building systems. However, the reason for reduction of manpower over the last 25 years has been the growth of living standards (Carol Sta, 2000). The Increasing development is leading the wages and is leading equal wages of construction workers with industrial workers and lead to ample and immediate changes in income of building work. All of these are confronted builders with new issues which are the acceptance of wage increases and increases in construction materials that the construction costs are increased very high. Modernization of making method leads to reducing construction costs. As a result, the share human resources in all things, the number of construction workers have become decreased increasingly. Also this industrialization is resisted in the face of rising prices by reducing the duration of their making products. Even in the case of some unit products could bring down the prices such as vehicles and industrial production floor. On the other hand, building system can avoid the cost of large facilities because time making is very short. Particularly in cases such as making new classrooms in a lightweight system isn't need to crane for montage (Jazbi, 2000). In building systems, due to the shortening making time building costs are also very low. In cases where the scheme is factory or office building, the run can be begin from the earlier stages. Also, relative to Housing projects, it could be completed in less time.

\section{NANO COVERINGS OF CONCRETE}

Nowadays, the use of new technologies in the concrete industry has been used in structures to improve the quality and the operation level of them. The Nano concrete is a new technology in this field, by using carbon nanotubes and Nano cement which are the most important materials in concrete production can be produced a concrete different than other concretes. Such as improvement of mechanical properties of concrete that containing particles of Nano compared with other concretes is because of filling pores with more concrete in scale of nanometers (Mohammadi Kia \& Razi Manesh Sorkhab, 2012).

Nano-silica is of particles which in concrete may improve its performance and more effectiveness in conserving energy in buildings. Nano-silica has created multi-purpose applications in the concrete. Among its uses can be cited to anti-wear properties, anti-slip and fireproofing and anti-reflection of concrete.

Mixing Nano-silica in dimensions 15 nanometer with cement paste has increased 0.36 the primary solidity of cement relative to paste without Nano-silica and is increased 28-day strength of concrete up to $13 \%$. Primary solidity is solidity that obtained during the first 7 days. With use of Nano silica in structural concrete used in roads, is more appropriate because of the higher primary solidity than ordinary concrete (Mohammadi Kia \& Razi Manesh Sorkhab, 2012). Another advantage of using Nano-silica is much better resistance and protection against chloride ions and sulfate (Abbaspour, 2010). coatings with Nano-materials are used in various industries to reduce energy costs, ideal insulation; reducing costs caused by corrosion of metals particularly types of building pipes, reduce lead emissions in the environment and protection of levels against moisture, mildew and fungi.

\section{COMPOSITES (CONCRETE FIBERS)}

Reinforced concrete with Glass fiber, GRC is a kind of concrete fibers and in fact is a kind of composite that by using the fiber amplifier inside the concrete mix are caused increasing in compressive strength and super tensile in this materials. Today, using types of composite concrete such as fibers concrete are possible in different industrial uses. Their uses in the developed countries of the world are common and have become popular in techniques of building industry. Fiber concrete in addition is a novel material with excellent properties in the field of alleviating the building weight, 
also is very impressive materials in mass production, especially in majority of made lightweight parts (Rudaki, 2012). By using the reinforcing fibers within the concrete mix, its tensile strength and compressive strength are super increased. This Composite formation has appropriate Integrity and continuity and the possibility of using concrete is provided as a flexible material for producing resistant surfaces with more curves. Fiber concrete also has a high energy absorption capability and under the impact loads cannot be easily torn apart. Historical evidence of this technology is thatch used in building construction. In fact, the fiber concrete is advanced type of this technology that new natural and synthetic fibers are successor of straw and cement are successor of mud that used in the thatch. distinctive features of fiber concrete are comprising low cost against high volume of operations, the possibility of fast and easy transportation, its use in most geographical regions, Formability due to the geometric shapes, high efficiency, very high resistance against tensile and compressive forces, energy absorption capability and stability against cracking finally.

Types of Concrete fiber are:

FRP: Fiber concrete using plastic fiber that is used in retrofitting structures;

AFRP: aramid fibers, the combination of concrete with fibers, and Nano technology.

GFRP: Concrete with carbon fiber and using carbon resins, such as: (carbon nanotubes)

Carbon is a wonderful element of nature and its numerous applications in human life well confirms this point. For example, steel that is one of the most important engineering alloys that obtained of dissolution about two percent of the carbon in iron. By varying the carbon content (As much just a few hundredths of a percent) could be achieved types of steel.

GRC: Glass fiber concrete

GRC: Fiber glass concrete or translucent concrete (Concrete passing the light).

\section{GLASS FIBER CONCRETE}

One of the most important concrete fibers is a glass fiber concrete that is also known as concrete passing the light. Today, concrete passing the light, as a new building material has the high usability. It is a combination of optical fibers and concrete particles and can be used as building blocks or prefabricated panels. Fibers because of their small size are mixed with concrete and is formed a combination of aggregation material. The major uses of concrete reinforced with glass fiber are in building facet, molding, sculptor, decorative array, restoration work, decor building, construction, drainage and cleans the bed. The structure of glass concrete isn't merely combined the two glasses and concrete, but also is a third new material in terms of the internal structure and outer surfaces is homogeneous completely. Glass fibers are causing the penetration of light into blocks. The most interesting phenomenon is showing shadows on the opposite side that is getting light. Also, the color of light that can be seen from behind the concrete is fixed. For example, if the green light shines behind the blocks, green shadows are seen in front of it. Thousands of light glass fibers together in parallel are placed between two main aspects of concrete blocks. Fiber ratio is very low and is about four percent of all the blocks. In addition, these fibers are mixed with concrete because of their small size and are become a structural component. So, the outer surface of the concrete remains homogeneous (Figure 5). 


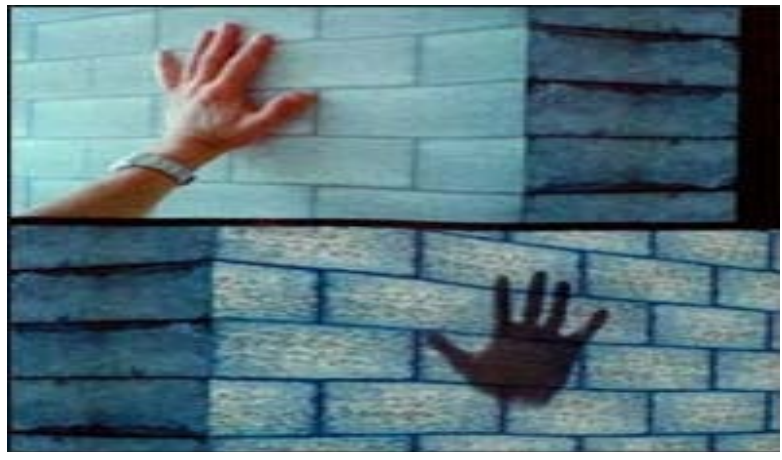

Figure 5. An example of glass fiber concrete

\section{CONCRETE AUTOCLAVE}

In the current situation the lightweight concrete or Heblex is the best material for small and large residential buildings, services, industrial and agricultural especially in seismic regions. Heblex is a mixture of sand, cement, lime and aluminum powder at a temperature of $200^{\circ} \mathrm{C}$ and pressure of 12 atmospheres that is baked in an autoclave and is sectioned to needed structural parts. Production of Heblex blocks in the construction industry is founded and started by deceased Ali Akbar Bajestani in 1988 in Iran. This product has special privileges than the other materials such as it is good thermal and sound insulation and is resistant against pressure, with the usual tools can be readily cut, can shaved to any shape, pierced and reshaped (Jodeiri-e-Abbasi \& Javanmard, 2012). In terms of structural components, making this product is done by mixing and baking raw materials. Approximately $60 \%$ of weight of raw materials is micronized silica aggregate with high purity of $80 \%$ and this amount of silica is non-replacement with other aggregates. Cement consumption is less than $100 \mathrm{~kg}$ per cubic meter. Used Aluminum oxide powder is with defined and special gradation.

\section{MONOLITH}

In recent years a lot of progress has been made in reducing the cost of building and in reducing weight of them and engineers have created different ideas in this field. For example: concrete, partitions, $3 \mathrm{~d}$ panels, Knauf and tile adhesive replaced with mortar of stone and etc. are one of the most important achievements of lightweight building and decreasing statistics of destruction and casualties caused by the earthquake as well as increase the speed of the implementation of the projects. The responsible of spreading the culture of the lightweight building is building engineers and designer. Light constriction can reduce 40 to $60 \%$ weight of buildings. Currently, new materials for building facades are entered the market that are replaced fast with materials such as brick, stone and glass, etc. Monolith facade is actually a combination of particles of marble, granite, quartz with its completely natural colors ingredients that are mixed in some process with special resins and lubricants (Figure 6).

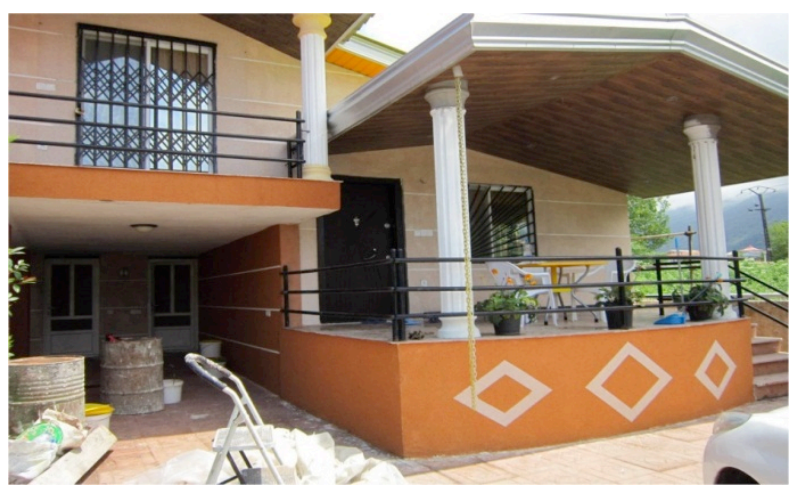

Figure 6. Image of sample run with monolith 


\section{FEATURES AND DIFFERENCES OF MONOLITH WITH STONE FACADES}

Beauty: to have natural colors and combining them with mix of several types of stones is converted to different colors and has 30 different colors coverage. Therefore, the openness of designer hands for a various design of facade are monolith benefits (Figure 7).

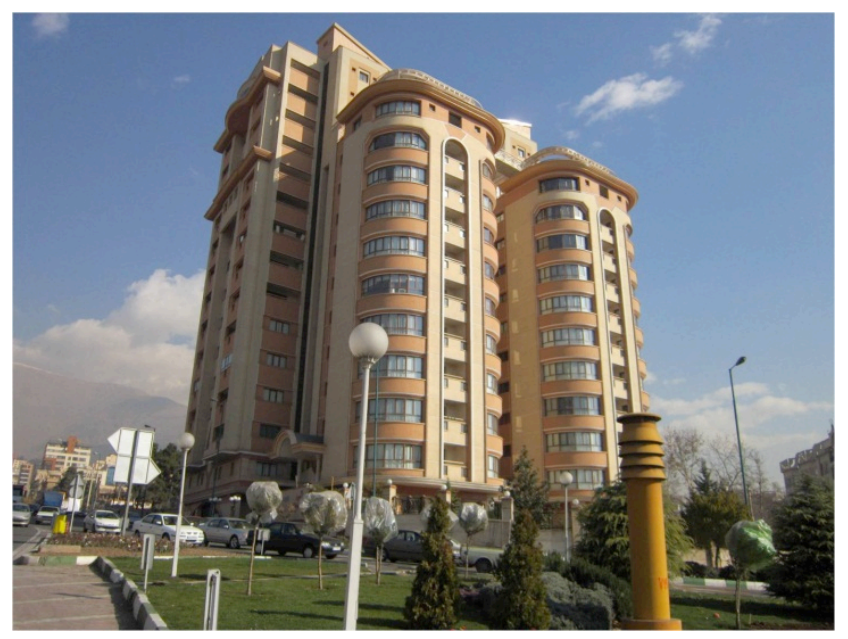

Figure 7. Mixing colors with the implementation of the monolith

Lightness: In terms of weight, monolith facade or aggregate is about 50 percent lighter than the stone facades. Buildings weight is one of the most important construction industries which will get a larger share of the earthquakes forces. As well the stone materials, brick, glass and composites are used in building facades because of their weight are at greater risk for detachment from the wall and thrown on the passage location of pedestrians. Excessive weight and volume of the rock and similar materials are demanded tougher infrastructure. In this regard, the buildings taller than four floors are less used of big stone materials and brick. So, the monolith facade is more placed in the spotlight. One of the biggest advantages of monolith coverage is being light weight than the other facade coverages. So that the monolith coverage weight used in per square meter is 3 to $4 \mathrm{~kg}$. Stone consumption per square meter is 80 to $100 \mathrm{~kg}$. Brick consumption per square meter is 20 to $40 \mathrm{~kg}$.

Noncombustible in fire: Monolith facade itself is not flammable and in case of fire does not ignite. It has been powder and flowed down. Other advantages are resistance in hot and cold weather conditions and humidity, anti-static and anti- Static electricity (dust proof), capability to dilute with water features and are environmentally friendly.

Other features are faster runtime and safety than the rock because of lightness and safety, ability to repair cheap, good moisture insulation against water infiltration due to become a nylon layer under the effect of having acrylic resin and silicon.

\section{RUN METHODS OF MONOLITH COVERAGE AND CONSUMING COSTS}

Run method of monolith facade: Materials are preparing in buckets of 20, 25 and $30 \mathrm{~kg}$. The run method is very minor amount of water added to the materials and then be performed by special trowel. The drying time is approximately 6 hours.

The cost of running the monolith coverage: in estimating the cost of the run monolith facade affects the simplification of work surface, the entire main area and the number of floors. 


\section{POLY STONE}

Poly stone can be considered as polymer stone with have all the capabilities of stone, could be produced in very large pieces and with desired flexibility and curvature. It is as hard as stone and with its beauty, but with this difference that isn 't made of stone. Its color variation is high. Poly stone has remarkable properties, particularly for use in bathrooms and kitchen. It can be put together the small pieces of poly stone and paste them with no space even to be fully integrated for miles. Poly stone can be created any type of flexibility and bending like plastics. Poly stone has at least 15 years useful life. After this time, its color changes that are created on contact with colorful food or scratches, corrosion and cracks can disappear with a simple sanding and again returns to the first image can be repaired. Its remnants have other uses. It is absolutely antibacterial. It is easily cleaned. It is also resistant against stains and crime. It has not much weight. Poly stone are used in making bathtub, WC, toilet and cover of different levels such as tables, kitchen cabinets and restaurants Because of being antibacterial.

\section{INDUSTRIAL RELATION WITH THE ECONOMIC COSTS OF BUILDING}

Planning for infrastructure of work construction Industry should be carried out so that with passing of times the created changes and developments has no negative effect on the efficiency and purpose. The most important tool for this job is main requirements of human. Because the lack of attention to this issue causes the non-ideal and unpleasant buildings before the end of their useful life will be demolished and rebuilt. Also this issue has needed to use materials, time and money and not only does not help to recycle materials but also produce waste (Kyoto protocol, 1997).

However, due to structural infrastructure and the principles governing the design and work construction, the previous sustainability in housing construction is guaranteed. So continuously control of quantity and quality are needed during useful life and most importantly, lower production costs and speed in construction.

This issue can be solved with genesis of building technologies day and run the specific techniques in work construction. Materials industrialization by running the specific methods can effectively help to the speed of work construction, preventing the stagnation in the national capital and bring down the cost.

\section{THE INTERACTION OF INDIGENOUS AND TRADITIONAL ARCHITECTURE WITH CONTEMPORARY ARCHITECTURE}

Because of some reason, the work construction industry has been commonplace in the community and plays a role in the promotion and development of sustainable architecture aligned with traditional construction. This is developed an idea in the architects mind that every action has a feedback. It is true that industrial architect with mass production of materials can be useful in the development of constructiveness and sustainable design, but it needed a massive movement of people and appropriate beds, Up to a culture where many years the mood and thoughts have been associated with it, changed or keep his place coordinated by traditional architecture. Thus, any change in its strategic process will bring challenges. Major attention of technical systems is on the small planning and traditional methods. Significant contribution of activities is moved based on two factors contracts. While by use of cultural thoughts of industrial work construction, the coordinated collaboration with designers and builders and craftsmen is created and grows. This strongly conflict with two-factor contracts. For this reason collection of technical system along with its subsidiaries as a serious and legal obstacle limits the development of the construction industry (Esmaeili-e-denarati \& aliborz, 2012). On the other hand, Due to the remarkable capacity and mass planning, the architectural designs more or less despite of flexibility and diversification are devoid of diversity and uniform. This is also another challenge.

Despite of industrialization by Day technologies of the world, the making system will be aligned with main elements such as human resource, machinery and etc. Its requirements are the presence of experts and consultants in the field. Such this possibility is very rare in Iran today, so the lack of presence of system design consultants are to some extent that this important issue is considered as a challenge. So the interaction of vernacular architecture with traditional culture required to fix these 
challenges in relation to architecture of the modern age. These actions could be based on the need to create industrial technology in Day architecture along with traditional or sustainable architecture. Architects or experts by studies the harmony of technologies both Modern and traditional with together and combining two ideas that are mixed with natural inspirations can be making culture. This as an progress in techniques of making building, making inexpensive and making smart are inserted to the society and its culture up to in this way of traditional architecture and decorative array are used in the architecture of the time or speed.

\section{INDUSTRIAL PRODUCTIONS CONTINUITY IN STRUCTURE AND ARCHITECTURE}

Referring to some of the advantages and disadvantages of industrial construction, it can be thought to continue in the future.

\section{ADVANTAGES}

1. The possibility of using management system and efficient project control;

2. Efficient use of materials for sustainable development;

3. Reduce energy consumption during the construction and operation for sustainable development;

4. Increase the speed of construction of building;

5. Ease of quality control at building Workshop;

6. Creating jobs for skilled labor;

7. Increase the useful life of the building and making lightweight.

\section{Disadvantages}

Height limitation at them, overlooking some of tastes, decreases in diversification due to massively and Uniform appearance of industrial making. At the present time, it is true that industry plays an important role in making mass and faster assembled buildings such as conex and prefabricated products or buildings with pre-stressed concrete, but will continue this materials manufacturing, these products will occur or industrial production will fade as time goes. Inferential answer to this question must be examined; researchers condemned this hypothesis that construction products defeats. Because progress is always ahead of retrogress in thoughts moves the minds to the endless places which it also opens a path that could take steps to promote. However, human needs has caused them always being in the thought of creativity or discovery. Every day have more varied design and architecture that these needs can be continued with creating new spaces, or by creating an incentive in different usage, interior decoration, light processing, making high or making smart. So the industrial age also to keep pace with this needs, are thriving and producing. Checking these factors underscore the continuity of industrial architecture. Nothing does not violate this principle, except poverty of economic resources or financial or shortage of primary elements and recycled materials of this production cycle.

\section{CONCLUSION}

Investigating the advantages and disadvantages of this type of making building ensure this issue that regardless of tradition, industrialism is obvious. Because, both tradition and industry in architecture, each one individually will have problems in the construction process and none of them alone does not meet the needs of society. Therefore, to eliminate the problems by industrial production method, it should be evaluated its usage and its capabilities in terms of preventing energy waste, materials and resources, high quality of building due to standardize the materials and modularization and on time distribution and installation time within the workshop location evaluation. Along with them, it should not be neglected training of skilled manpower in the field of machinery and equipment. Until the promising points in combining industry and tradition is identified and reinforced in order to provide its progress to improve the quality and quantity of new material in the not too distant future. 


\section{REFERENCES}

Abbaspour M, 2010. Study of increasing the Nano-silica in concrete. The first National Conference and the fourth National Conference on Conference Institute of Higher Education Khavaran, 1-4-6pp.

Bashiri M, 2012. Affordable housing from dream to reality. Journal of architecture and culture. 48 (14): 10-16.

Carol Sta TS, 2000. Design trends in prefabrication of building. Translated by Alireza Jazbi. Khak publication. Esfahan, 256pp.

Esmaeili-e-denarati E, aliborz M, 2012. Cited in scientific works: industrialization of building a strategy to use new technologies. Publications of ministry of Culture and Islamic Guidance 8971: 223226.

Jodeiri-e-Abbasi M, 2012. University Course notes.

Helman L, 2002. The Architecture alphabet. Translated by Mohammad Ahmadinezhad. Khak publication. Esfahan, 192pp.

Mohammadi Kia M, Razi Manesh Sorkhab B, 2012. Cited in scientific works: Nano technology and its role in hardening concrete properties. Publications of ministry of Culture and Islamic Guidance 8971: 122-132.

http://www.alandamir.ir/page.php?menu=10\&menuName, Production and running new coatings (MONOLITH). 